\title{
Optimization of the threshold pump power of a photonic crystal nanolaser, experiment and theory
}

Mathiesen, K. S.; Rasmussen, T. S.; Yu, Y.; Sakanas, A.; Semenova, E.; Yvind, K.; Mørk, J.

Published in:

Proceedings of SPIE

Link to article, DOI:

$10.1117 / 12.2520896$

Publication date:

2019

Document Version

Publisher's PDF, also known as Version of record

Link back to DTU Orbit

Citation (APA):

Mathiesen, K. S., Rasmussen, T. S., Yu, Y., Sakanas, A., Semenova, E., Yvind, K., \& Mørk, J. (2019).

Optimization of the threshold pump power of a photonic crystal nanolaser, experiment and theory. In P. Cheben, J. Ctyroky, \& I. Molina-Fernandez (Eds.), Proceedings of SPIE (Vol. 11031). [110310G] SPIE - International Society for Optical Engineering. Proceedings of SPIE - The International Society for Optical Engineering Vol. 11031 https://doi.org/10.1117/12.2520896

\section{General rights}

Copyright and moral rights for the publications made accessible in the public portal are retained by the authors and/or other copyright owners and it is a condition of accessing publications that users recognise and abide by the legal requirements associated with these rights.

- Users may download and print one copy of any publication from the public portal for the purpose of private study or research.

- You may not further distribute the material or use it for any profit-making activity or commercial gain

- You may freely distribute the URL identifying the publication in the public portal 


\section{Optimization of the threshold pump power of a photonic crystal nanolaser, experiment and theory}

K. S. Mathiesen, T. S. Rasmussen, Y. Yu, A. Sakanas, E. Semenova, et al.

K. S. Mathiesen, T. S. Rasmussen, Y. Yu, A. Sakanas, E. Semenova, K. Yvind, J. Mørk, "Optimization of the threshold pump power of a photonic crystal nanolaser, experiment and theory," Proc. SPIE 11031, Integrated Optics: Design, Devices, Systems, and Applications V, 110310G (26 April 2019); doi: 10.1117/12.2520896

SPE. Event: SPIE Optics + Optoelectronics, 2019, Prague, Czech Republic 


\title{
Optimization of the threshold pump power of a photonic crystal nanolaser, experiment and theory
}

\author{
K. S. Mathiesen, T. S. Rasmussen, Y. Yu, A. Sakanas, E. Semenova, K. Yvind, and J. Mørk \\ Technical University of Denmark, DTU Fotonik, Ørsteds Plads, Kgs. Lyngby, Denmark
}

\begin{abstract}
Here we experimentally characterize photonic crystal nanolasers where the first endhole of the mirror has been systamatically shifted. FDTD simulations of similar passive cavities are done in order to find the expected evolution of the quality factor. We find that the predicted increase in the quality factor of the equivalent passive cavities leads to a decrease in the threshold power of the active nanolasers as expected. The maximum output power for varying endhole shifts has also been investigated and shifting the holes to optimize quality factor leads to lower maximum output power, when measuring from the top. The mirror of the photonic crystal cavity is further investigated as the mirror phase and penetration depth into the mirror are determined as a function of the endhole shift.
\end{abstract}

Keywords: Nanolasers, Photonic crystals, FDTD, Cavity mirror phase, Optically pumped lasers, Quantum dot lasers, Lasers, Line defect lasers

\section{INTRODUCTION}

The development of ultra-small and efficient laser sources is an enabling technology for realizing optical on-chip interconnects. ${ }^{1}$ A key figure of merit is the threshold power of the nanolaser. A line defect cavity formed by omitting a number of holes in a photonic crystal membrane with embedded quantum dot gain material shows promise as a candidate for realizing lasers with small mode volumes and low threshold powers, while allowing direct modulation at several gigabits per second. ${ }^{2}$ It has been shown that shifting the first in-line hole of the mirrors in a passive structure increases the cavity quality factor significantly, ${ }^{3}$ and allowing multiple holes to be shifted further increases it. ${ }^{4}$ So far the only systematic investigations of such hole shifts have been carried out by measuring the quality factor of passive devices. From a conventional laser rate-equation model, ${ }^{5}$ it is expected that an increase in the quality factor corresponds to a decrease in the threshold power of the laser. Here we report a systematic investigation of line defect cavity lasers with varying hole shifts using an out-of plane pumping and collection setup. From the experimental characterization the quality factor and the threshold pump power can be extracted. ${ }^{6}$ The dependence of the maximum power output on the hole shift is also investigated. The experimental results are compared to full 3D finite difference time domain (FDTD) simulations of the corresponding passive cavities. Since the termination of the line-defect waveguide functions as an effective Bragg mirror, FDTD calculations are used to calculate the mirror phase and penetration depth using an effective Fabry-Perot cavity model, ${ }^{7}$ in order to compare the hole-shifted and regular structures. These numerical results are compared to results of the experimental characterization.

\subsection{Sample design}

The photonic crystal lasers are fabricated in a $250 \mathrm{~nm}$ thick InP membrane with three layers of embedded InAs quantum dots, see figure 1a. The membrane is above silica on a silicon substrate. All the fabricated cavities are made by omitting seven holes in the photonic crystal leading to a so called L7 line-defect laser. All the fabricated cavities has a period of $a=0.438 \mathrm{um}$, and varying hole radii that are determined from SEM images. The equivalent passive cavit without endhole shifts has a numerical Q-factor of 60000 . By shifting the first hole of the in-line mirror the Q-factor can be improved drastically, and samples are fabricated with (0-0.4)a shifts in increments of $0.05 \mathrm{a}$, see figure $1 \mathrm{~b}$. This allows us to systematically investigate how the laser threshold and output power depends on the hole shift.

Further author information: (Send correspondence to K. S. M.)

K.S.M.: E-mail: krsma@fotonik.dtu.dk, Telephone: +45 45256871

Integrated Optics: Design, Devices, Systems, and Applications V, edited by Pavel Cheben, Jiří Čtyroký, Iñigo Molina-Fernández, Proc. of SPIE Vol. 11031, 110310G · C 2019 SPIE · CCC code: $0277-786 \mathrm{X} / 19 / \$ 18 \cdot$ doi: $10.1117 / 12.2520896$

Proc. of SPIE Vol. 11031 110310G-1 


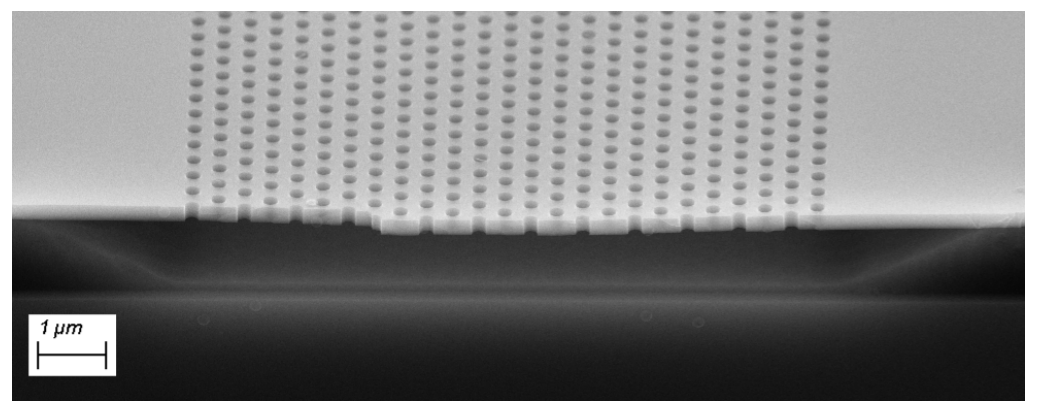

(a) The InP membrane with the embedded quantum dots.

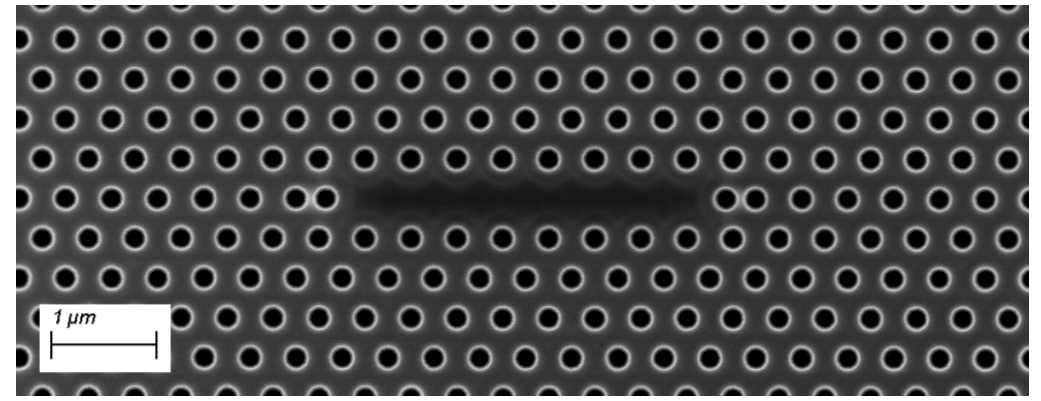

(b) An L7 cavity with the first endholes shifted by 0.35 a.

Figure 1: SEM images of the samples.

\subsection{Rate equations and the threshold power dependence on quality factor}

Starting from rate equations of the carrier $N$ and photon density $N_{p}$ in the laser cavity it is possible to determine how the threshold power, $P_{t h}$, of the laser is related to the quality factor $Q$. An analytical solution is obtained for steady state if an assumption of linear gain is made, i.e. $g(N)=\frac{g_{0}}{N_{t r}}\left(N-N_{t r}\right)$, where $N_{t r}$ is the transparency carrier density. The derivations can be found in A. Ultimately

$$
P_{t h} \propto \frac{1}{\tau_{p}} \propto \frac{1}{Q},
$$

where $\tau_{p}$ is the photon lifetime in the cavity. It follows then that an increase in quality factor of the cavity corresponds to a lower threshold power of the laser.

\section{MEASUREMENT SETUP}

The experimental setup can be seen in figure 2. The sample is pumped by a $300 \mathrm{~mW}$ CW $1480 \mathrm{~nm}$ laser diode through a $50 \times, 0.65 \mathrm{NA}$ objective. Scattered light from the cavity as well as reflected pump light is then collected from the top and passes through a fiber based WDM that filters out the pump light $<1510 \mathrm{~nm}$ before it is collected in an optical spectrum analyser. A fiber based attenuator in the line allows the input power to be swept in order to measure input-output curves of the nanolasers.

\section{EXPERIMENTAL RESULTS}

Two samples for a total of five groups with hole shifts from (0-0.4)a has been measured. Their input-output curves can be seen in figure $3 \mathrm{a}$ and $3 \mathrm{~b}$ in linear and logarithmic scales respectively. The output power is seen to fluctuate between the groups, also for similar radii. The radii are measured using SEM and on the same sample only one group has been measured. As such there might be slight differences here. Further there are local variations in the hole sizes and thus the photonic crystal environment of different laser cavities in the groups are not completely identical. 


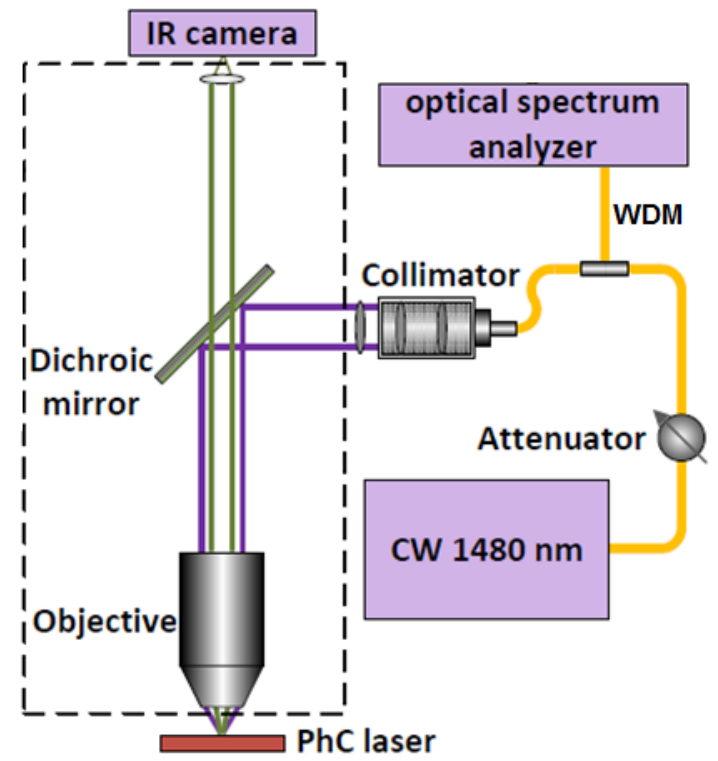

Figure 2: The experimental setup used for characterization of the nanolasers. A $1480 \mathrm{~nm} \mathrm{CW}$ diode is used for optical pumping of the sample. The collected light is sent through a fiber based WDM to filter out the pump light $<1510 \mathrm{~nm}$ and collected by an optical spectrum analyser.

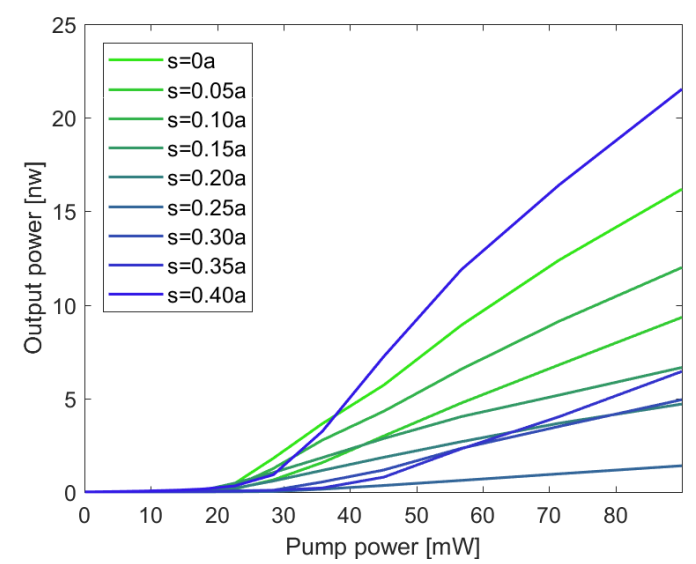

(a) Linear scale.

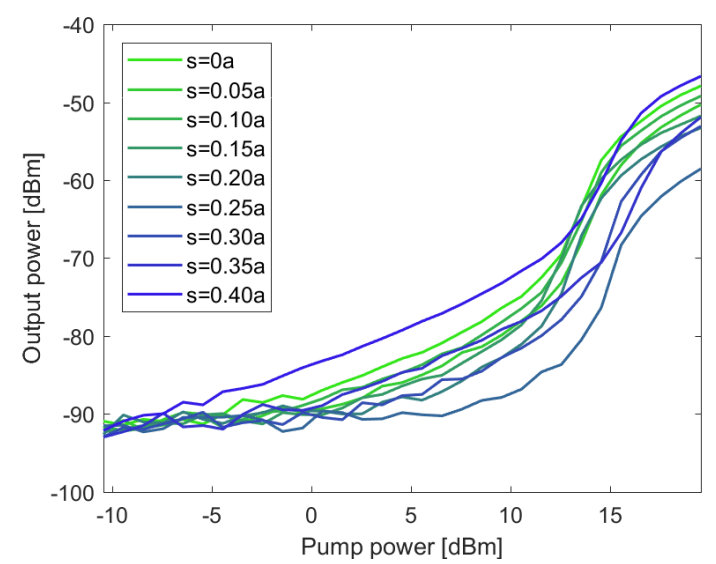

(b) Logarithmic scale.

Figure 3: Experimental input-output curves measured for the $r_{1}=95.7 \mathrm{~nm}$ group when varying the endhole shifts. The characteristic s-shape of a laser is noticeable in the logarithmic plot when the threshold is reached.

The threshold powers are determined from the linear input-output curves by fitting a second order polynomial to the area around the maximum value the second derivative. The extracted threshold powers can be seen in figure 4a. Similarly to the output powers, the threshold powers also fluctuate between the groups. To investigate the dependence of the threshold power as a function of the hole-shift it is then more instructional to look at the normalized powers, which can be seen in figure $4 \mathrm{~b}$. Here the numerical results for similar passive cavities are also superimposed. The same trend is seen in both the simulations and the experimental results. While the numerical result promise even higher Q-factors as we near the optimum hole shift, this may not have been reached due to fabrication imperfections. Similarly the increase in threshold power after the optimum endhole shift is not as drastic in the experiments.

The maximum output powers are reached at maximum pump power and the measured values can be seen in figure $5 \mathrm{a}$ and normalized in figure $5 \mathrm{~b}$. The output power shows a less clear trend and highlights how the 


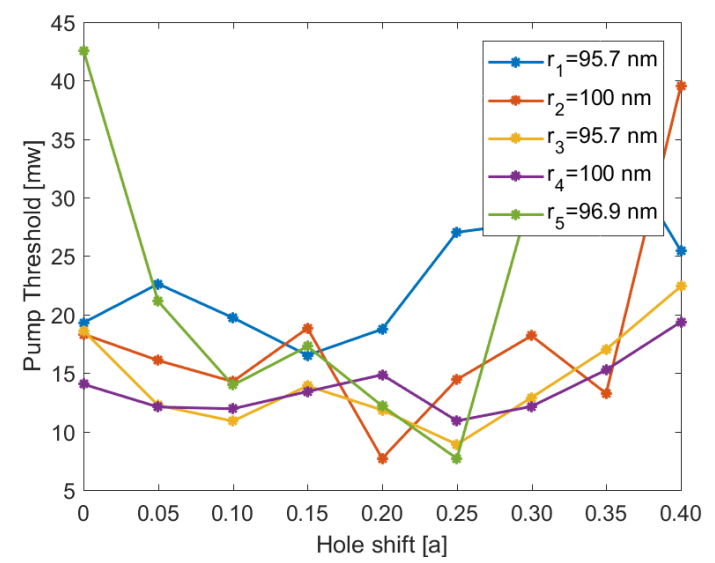

(a)

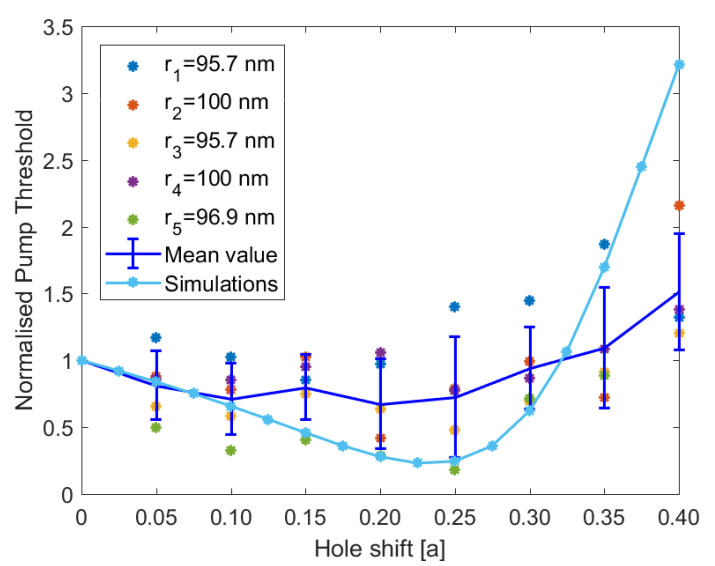

(b)

Figure 4: (a) Threshold pump powers extracted from the measurements. The structures have different hole radius ranging from (95-100) $\mathrm{nm}$ (b) Normalized threshold pump powers and comparison with results from simulations on an equivalent passive cavity. The experimental results show a similar trend as the endhole shift is varied.

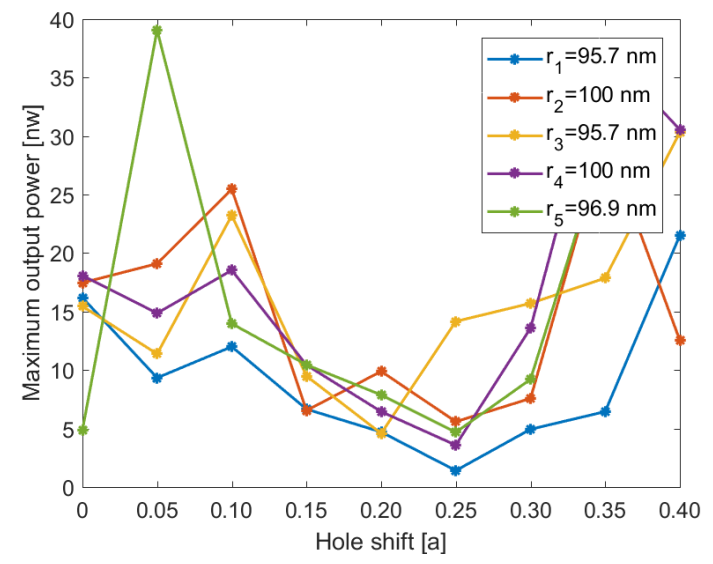

(a)

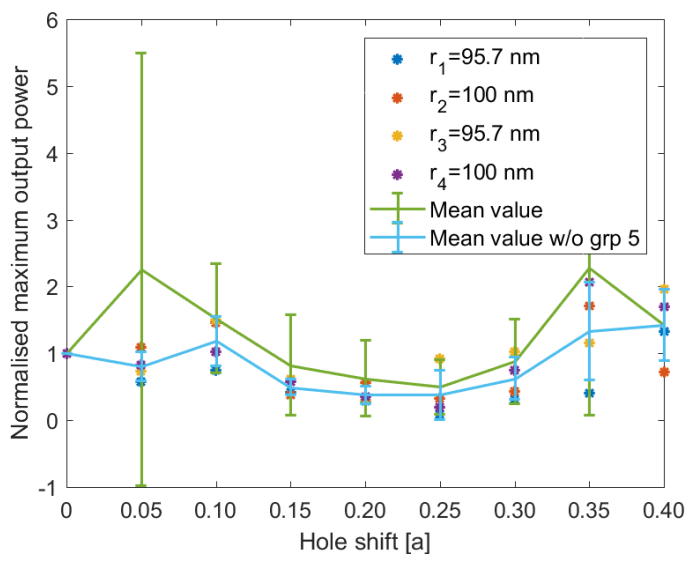

(b)

Figure 5: (a) Maximum output powers for each of the laser cavities. As can be seen the last group measured deviates in behaviour having a high maximum output power for $0.05 \mathrm{a}$ shift compared to 0 shift. (b) Normalized maximum output power. The group that deviates in behaviour is excluded from the plot and mean calculations both with, and without this group is shown.

behaviour changes across different groups. The maximum output power seems to drop following the same trend as the threshold power, and then increases for the shifts after the optimum Q. However it also appears that an endhole shift of 0.10a is a local maximum for the maximum output power. As found by Akahane et al. ${ }^{3}$ the cavity fields can be Fourier transformed and when doing an inverse transform of only the part of the field within the light cone the shifting of the first endhole results directly in less scattering which could explain why the output power first decreases.

\section{NUMERICAL RESULTS}

The passive cavities can be simulated in the commercial FDTD software Lumerical. The resulting field profiles from an example simulation for an L7 cavity with an endhole shifted $0.25 \mathrm{a}$ can be seen in figure 6a. The simulation tools have also been used to simulate longer and shorter cavities with the same varying endhole shifts. 


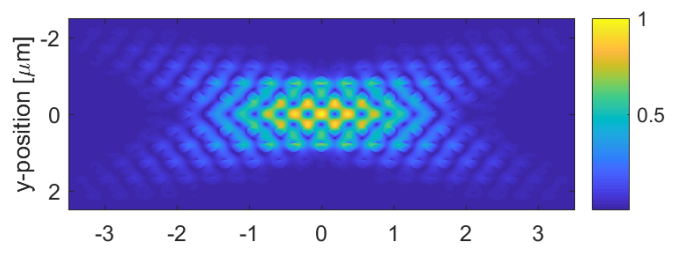

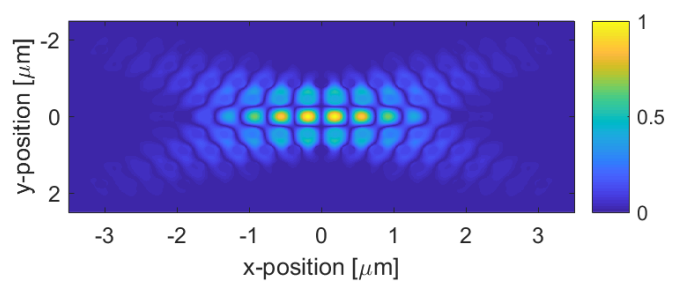

(a)

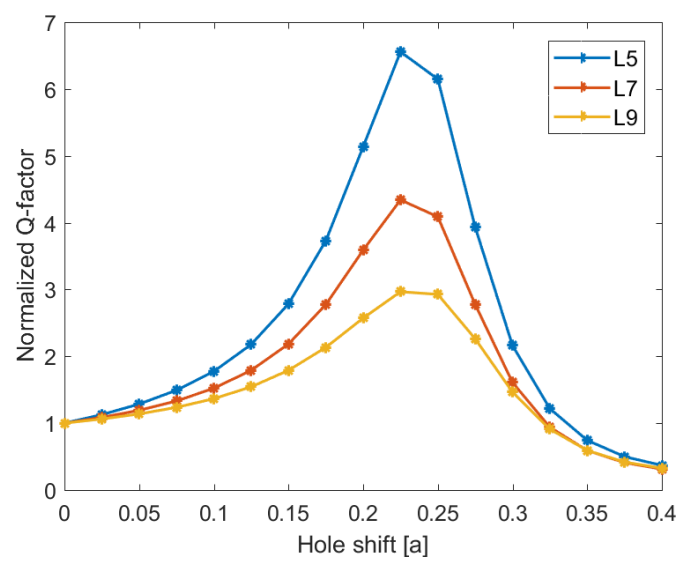

(b)

Figure 6: FDTD simulations on passive cavities. (a) Electric (top) and Magnetic (bottom) field profiles of the resonant mode for an L7 cavity. (b) Normalized quality factors of L5, L7 and L9 cavities as the endhole is shifted.

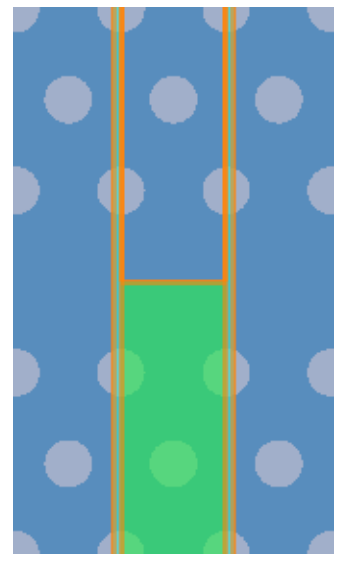

(a)

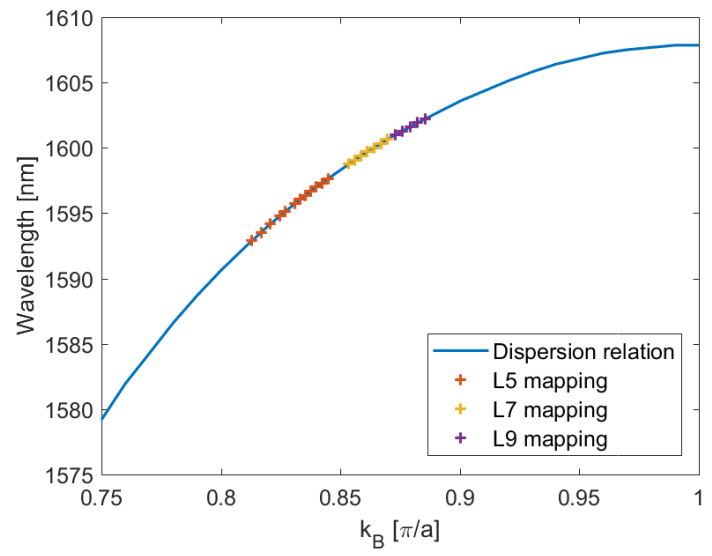

(b)

Figure 7: FDTD simulations on a passive waveguide. (a) Setup for determining the dispersion relation of the waveguide. A single period in the x-direction with Bloch boundary conditions is enough. (b) The resonance wavelengths of the L5, L7 and L9 cavities mapped onto the dispersion relation of the waveguide.

The normalized quality factors from these simulations can be seen in figure $6 \mathrm{~b}$, where they all carry the same trend. All the simulations are performed on passive membranes with hole radius of $99 \mathrm{~nm}$, a period of $438 \mathrm{~nm}$ and a membrane thickness of $250 \mathrm{~nm}$.

To calculate the dispersion diagram of the photonic crystal a simulation with periodic Bloch boundary conditions have been setup, figure $7 \mathrm{a}$ and the Bloch wavenumber $k_{B}$ is swept to obtain the dispersion diagram seen in figure $7 \mathrm{~b}$. The resonance wavelengths from the simulations of the passive cavities can then be mapped onto this curve to extract their corresponding wavevector to be used for phase calculations which is also seen in figure $7 \mathrm{~b}$.

\subsection{Phase calculations}

To calculate the mirror phase from the photonic crystal an effective Fabry-Perot resonator model is used. ${ }^{7}$ The length of this cavity is defined as $L_{e f f}=L+2 L_{p}$, where $L$ denotes the physical cavity length and $L_{p}$ is the penetration depth, which is further discussed in the next section. The resonance condition leads to the following phase relation 


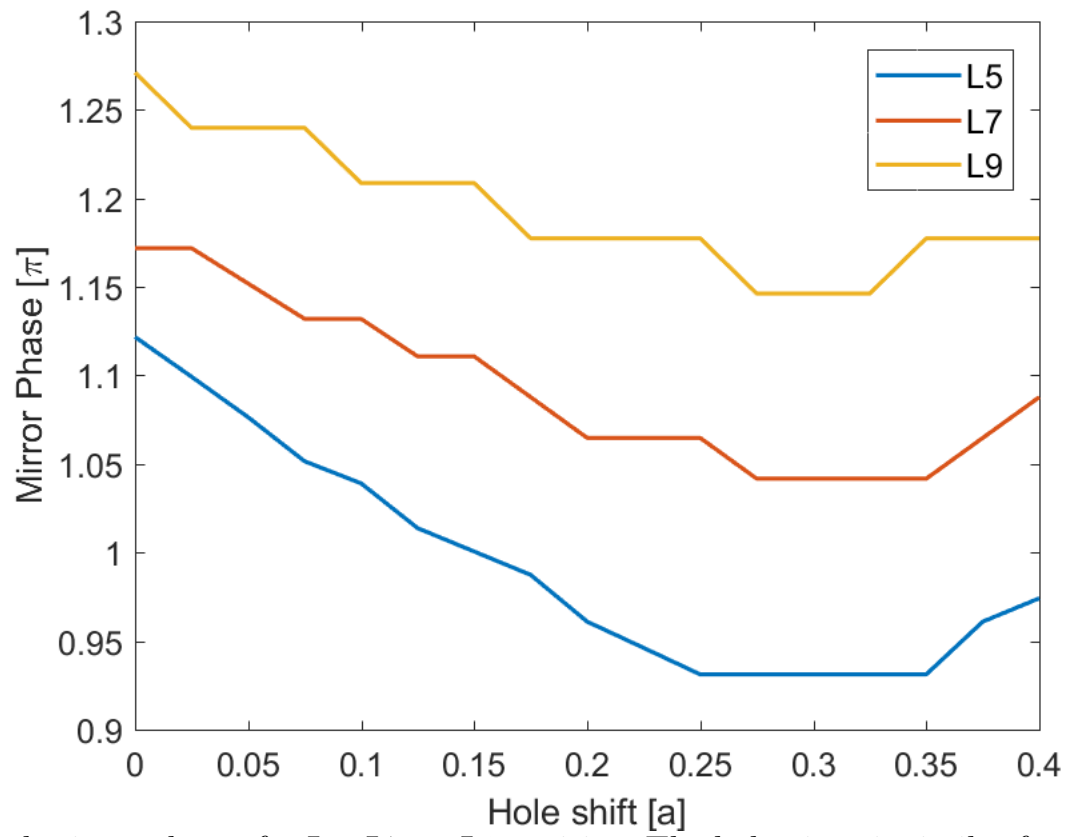

Figure 8: Calculated mirror phases for L5, L7 an L9 cavities. The behaviour is similar for all three cavity lengths however the absolute phase change increases as the cavity length increases.

$$
\pi p=\phi(\lambda)+k_{B} L
$$

where $\mathrm{p}$ is the number of oscillations in the magnetic field of the standing wave along the cavity, $\phi(\lambda)$ is the mirror phase and $k_{B}$ is the Bloch wavenumber. For all the phase calculations the length is defined as $L=(N+1) a$ and the number of oscillations is given as $p=N+1$. This way, the changes induced by shifting the endholes are absorbed into the mirror phase. The results can be seen in figure 8 for three different cavity lengths, L5, L7 and L9. The mirror phase is seen to vary as the endhole is shifted and the variation is similar for the three cavity lengths. The absolute phase change of the mirrors increase as the cavity length increases.

\subsection{Penetration depth}

Next the penetration depth, $L_{p}$ into the photonic crystal mirror is investigated. For the effective Fabry-Perot model the photonic crystal mirror can be interpreted with conventional grating mirror theory. For a conventionaal Bragg mirror, the penetration depth is related to the mirror phase as

$$
L_{p}=-\left.\frac{\lambda_{0}^{2}}{4 \pi n_{g}} \frac{\mathrm{d} \phi}{\mathrm{d} \lambda_{0}}\right|_{\lambda_{0}}
$$

where $\lambda_{0}$ is the resonance wavelength, $n_{g}$ is the group velocity determined from the dispersion relation, and $\mathrm{d} \phi /\left.\mathrm{d} \lambda_{0}\right|_{\lambda_{0}}$ is the phase change as a function of cavity wavelength. ${ }^{5}$ Here, this is determined for an L7 cavity by including simulations on L5 and L9 cavities and extracting the theoretical result. The calculated penetration depths as a function of endhole shift can be seen in figure $9 \mathrm{a}$. The penetration depth is seen vary around $1 \mathrm{um}$, and the result could benefit from a better resolution for the calculation on the mirror phase. The penetration depth may also be studied directly from the numerical simulations by a 1D H-field monitor along the center line. This can be seen in figure 9b for an L7 cavity as the endhole shift is varied. The H-field intensity is displayed for $x=L / 2=(N+1) a / 2$ and further away from the cavity center. The calculated penetration depths are mapped onto the simulated results. It appears that the calculated penetration depth to some degree reflects both the intensity of the field at the border and the decay away from the border of the effective Fabry-Perot cavity. 


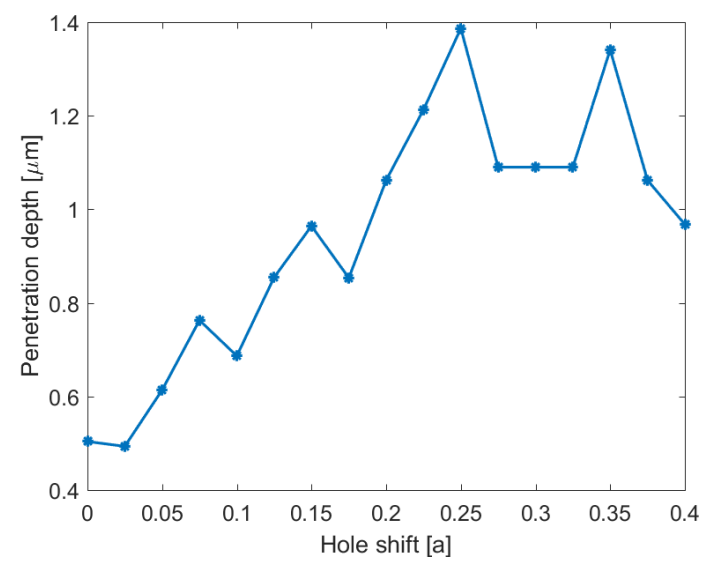

(a)

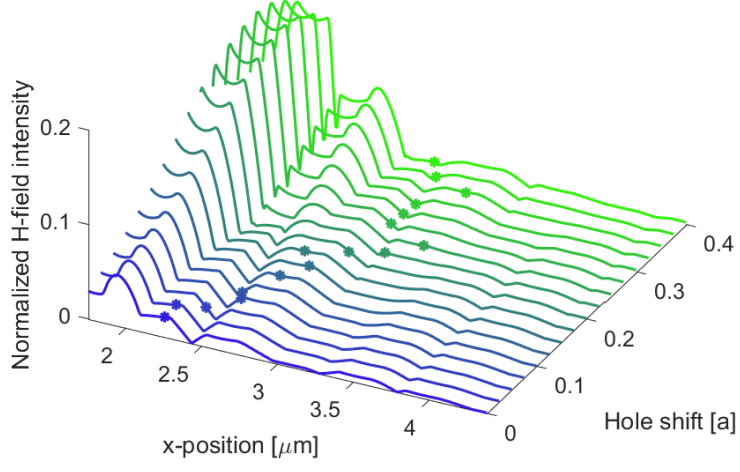

(b)

Figure 9: (a) Penetration depth calculated from the mirror phase extracted from simulations. (b) Visualization of simulated H-field penetration into the mirror as the endhole shift is varied. The x-axis starts at $L=(N+1) a / 2$, corresponding to the edge of the cavity. The dots are the calculated penetration depth seen in (a) mapped onto the simulated results.

\section{CONCLUSION}

Samples with photonic crystal nanolasers with systematic variation of the first endhole of the photonic crystal mirror have been fabricated and characterized to determine if the predicted increase in Q-factor of similar passive cavities are directly translatable into lower threshold pump powers of the active nanolasers. In general this was confirmed though less decrease in the threshold pump power is observed experimentally than what is predicted from simulations. All optimized photonic crystal cavities become more susceptible to fabrication imperfections and this could explain why the predicted thresholds are not obtained, as there might be an upper limit in reachable Q. The maximum output power of the nanolasers has also been measured and the output power becomes slightly lower as the optimum endhole shift is reached to then increase for larger than optimum endhole shifts. Here the light is collected vertically and a higher quality factor directly leads to a lower rate of vertical scattering. It would be interesting to repeat the experiment with in-plane light collection, which would however require samples that have active material only in the cavity region. Secondly more simulations and further rate equation investigations could determine how a higher quality factor impacts the photon densities to further link the quality factor and the maximum output power and explain the decrease in maximum output power when endholes are shifted towards the optimum value.

An effective Fabry-Perot model has been applied to the simulations of passive cavities in order to calculate the phase of the photonic crystal mirror and the penetration depth into the mirror. The phase of the mirrors change as first endhole is shifted and for all cavity lengths it first decreases until a shift of 0.275 a and increases again for larger shifts. It should however be noted that as the mirror phase is directly dependent on the effective cavity length $L$ it is clear that the definition of $L$ is very important and through the literature different definitions can be found. ${ }^{6-8}$ Further investigations should be done in order to distinguish these. Finally the penetration depths have been determined for the simulated L7 cavities both by looking at the 1D magnetic field profiles and

by calculations from the mirror phase. The calculated penetration depths appear to capture the decay observed in the field profiles to some degree.

\section{APPENDIX A. DERIVATION OF $Q$ AND $P_{T H}$ CORRELATION}

The following derivations are based on. ${ }^{5}$ The rate equations for carrier, $N$ and photon, $N_{p}$ densities can be described by the sum of rates into, minus the sum of rates out of the respective reservoirs. Further, any natural decay can be described by the carrier density and a decay time, e.g. $R_{s p}=N / \tau_{r}$. 


$$
\begin{gathered}
\frac{\mathrm{d} N}{\mathrm{~d} t}=\frac{\eta_{i} P_{i}}{h \nu V}-\left(\frac{N}{\tau_{r}}+\frac{N}{\tau_{n r}}\right)-v_{g} g N_{p}, \\
\frac{\mathrm{d} N_{p}}{\mathrm{~d} t}=\left[\Gamma v_{g} g-\frac{1}{\tau_{p}}\right] N_{p}+\Gamma \beta \frac{N}{\tau_{r}} .
\end{gathered}
$$

The generation of carriers by optical pumping can be described by the pumping power, $P_{i}$, the energy, $h \nu$ of the pumping photons, the volume of the active area, $V$ and the injection efficiency, $\eta_{i}$ that describes the amount of injected photons to successfully create carriers in the active region. Carriers are lost by spontaneous emission, $N / \tau_{r}$, non-radiative emission, $N / \tau_{n r}$ and stimulated emission $v_{g} g N_{p}$. The stimulated emission is described by the group velocity of the photons in the medium, $v_{g}$, the gain, $g$ which will be described below and the photon number $N_{p}$. For the photon density, the creation terms are from stimulated, $\Gamma v_{g} g N_{p}$ and spontaneous emission into the mode $\Gamma \beta \frac{N}{\tau_{r}}$, where $\Gamma=V / V_{p}$ is the confinement factor, simply the ratio between the active volume and the volume occupied by the photons (mode volume). The $\beta$-factor describes the ratio of spontaneous emission that contributes photons to the mode to the total spontaneous emission. The loss of photons in the cavity is described by the lifetime $\tau_{p}$. The gain is dependent on the electron density $g=g(N)$, and is often decribed with a logarithmic dependency. Here we continue the derivations with an assumption of a linear gain model as this allows to arrive at an analytical correlation between quality factor and threshold power. Then,

$$
g(N)=\frac{g_{0}}{N_{t r}}\left(N-N_{t r}\right),
$$

where $N_{t r}$ is the transparency carrier density, describing the carrier density needed for the gain material to appear transparent to an incoming electric field, ie. for an electric field passing through a length of gain material $g\left(N_{t r}\right)$ the electric field intensity remains unchanged.

The threshold power of the laser is defined as the point where the spontaneous emission has clamped and all further input power goes to stimulated emission. Below threshold the photon density is very low, and it is often a reasonable approximation to use $N_{p} \approx 0$. Then, for steady state at threshold, equation 4 a reduces to

$$
\frac{\eta_{i} P_{t h}}{h \nu V}=\left[\frac{1}{\tau_{r}}+\frac{1}{\tau_{n r}}\right] N_{t h}
$$

where $N_{t h}$ denotes the carrier density at threshold. At steady state threshold the gain must exactly counter the photons lost and

$$
g_{t h}=\frac{g_{0}}{N_{t r}}\left(N_{t h}-N_{t r}\right)=\frac{1}{\tau_{p} \Gamma v_{g}} .
$$

Finally combining equations 6 and 7 the threshold power can be found as

$$
P_{t h}=\frac{h \nu V}{\eta_{i}}\left[\frac{1}{\tau_{r}}+\frac{1}{\tau_{n r}}\right]\left[\frac{N_{t r}}{\tau_{p} \Gamma v_{g} g_{0}}+N_{t r}\right]
$$

from which it is clear that

$$
P_{t h} \propto \frac{1}{\tau_{p}} \propto \frac{1}{Q}
$$

\section{ACKNOWLEDGMENTS}

The author thanks the Villum Foundation for funding through the NATEC (NAnophotonics for Terabit Communications) Centre of Excellence (grant no. 8692). 


\section{REFERENCES}

[1] Miller, D., "Device Requirements for Optical Interconnects to Silicon Chips," Proceedings of the IEEE 97, 1166-1185 (jul 2009).

[2] Takeda, K., Sato, T., Shinya, A., Nozaki, K., Kobayashi, W., Taniyama, H., Notomi, M., Hasebe, K., Kakitsuka, T., and Matsuo, S., "Few-fJ/bit data transmissions using directly modulated lambda-scale embedded active region photonic-crystal lasers," Nature Photonics 7, 569-575 (jul 2013).

[3] Akahane, Y., Asano, T., Song, B.-S., and Noda, S., "High-Q photonic nanocavity in a two-dimensional photonic crystal," Nature 425, 944-947 (oct 2003).

[4] Maeno, K., Takahashi, Y., Nakamura, T., Asano, T., and Noda, S., "Analysis of high-Q photonic crystal L3 nanocavities designed by visualization of the leaky components," Optics Express 25, 367 (jan 2017).

[5] Coldren, L. A., Corzine, S. W., and Mašanović, M. L., [Diode Lasers and Photonic Integrated Circuits], John Wiley \& Sons, Inc., Hoboken, NJ, USA (mar 2012).

[6] Xue, W., Yu, Y., Ottaviano, L., Chen, Y., Semenova, E., Yvind, K., and Mork, J., "Threshold Characteristics of Slow-Light Photonic Crystal Lasers," Physical Review Letters 116, 063901 (feb 2016).

[7] Lalanne, P., Sauvan, C., and Hugonin, J., "Photon confinement in photonic crystal nanocavities," Laser 83 Photonics Review 2, 514-526 (dec 2008).

[8] Okano, M., Yamada, T., Sugisaka, J., Yamamoto, N., Itoh, M., Sugaya, T., Komori, K., and Mori, M., "Analysis of two-dimensional photonic crystal L-type cavities with low-refractive-index material cladding," Journal of Optics 12, 075101 (jul 2010). 